\title{
Two-section Fiber Optic Raman Polarizer
}

\author{
Sergey Sergeyev, Sergei Popov
}

\begin{abstract}
We report on a theoretical study of polarization impairments in periodically spun fiber Raman amplifiers. Using the Stochastic Generator approach we have derived averaged equations to calculate polarization dependent gain and meansquare gain fluctuations. We show that periodically spun fiber can work as a Raman polarizer but it suffers from increased polarization dependent gain and gain fluctuations. Unlike this, application of a depolarizer can result in suppression of polarization dependent gain and gain fluctuations. We demonstrate that it is possible to design a new fiber Raman polarizer by combining a short standard fiber with properly chosen parameters and a long periodically spun fiber. Such a polarizer provides almost the same polarization pulling for all input signal states of polarization and so has very small polarization dependent gain.
\end{abstract}

Index Terms - Optical fiber amplifiers, Raman scattering.

\section{INTRODUCTION}

$\mathrm{P}$ olarization mode dispersion (PMD) and polarization dependent gain (PDG) are among major factors limiting the progress in further increase of overall capacity of the next generation of optical networks based on distributed fiber Raman amplifier (FRA). PMD is caused by varying group velocities for the pulses with different states of polarization (SOPs) and so leads to pulse broadening [1-3]. Varying signal SOPs at the input of fiber Raman amplifier leads to uncontrollable output gain variation. Maximum of the gain variation is defined as polarization dependent gain (PDG) [48]. Traditional PMD suppression technique is based on spinning the fiber periodically and results in PMD suppression below $0.04 \mathrm{ps} / \mathrm{km}^{1 / 2}[2,3]$. However, this PMD suppression is accompanied with a simultaneous increase in the Raman PDG [5-8]. The new approach developed by Sergeyev et al. $[7,8]$ have shown that it is possible to simultaneously mitigate both PDG and PMD by using a fiber with a particular spin profile or, more specifically, a two-section fiber ('two-section

Manuscript received August 25, 2011. This work is supported in part by European Union program FP7 PEOPLE-2009-IEF (grant 253297).

Sergey Sergeyev is with Photonics Research Group, School of Engineering \& Applied Science, Aston University, Birmingham, B4 7ET, UK (corresponding author, phone: +44-121-204-3524; e-mail: ssergeyev@wit.ie, sergey.sergeyev@gmail.com).

Sergei Popov is with Royal Institute of Technology (KTH), Department of Microelectronics and Applied Physics, Electrum 229, SE-164 40 Kista, Sweden (e-mail: sergeip@kth.se). approach') in which the first section has no spin and the second one is periodically spun. A new approach based on using anisotropy of Raman amplification (Raman polarizer) to repolarize and amplify signal rather than to depolarize it has been recently developed by Martinelli et al. [9], Kozlov et al. [10] and Ursinini et al. [11]. In this approach, arbitrary at the input, signal SOP with low input power is attracting to the pump SOP with high input power (polarization pulling) and so is stabilizing [9-11]. In view of the lack of information about the gain fluctuation caused by a random birefringence, the characterization of different PMD and PDG mitigation schemes is incomplete. In this paper, we fill this knowledge gap by deriving equations and calculating mean-square gain fluctuations for different cases of application of a depolarizer, two-section approach and a Raman polarizer. The results show that the two-section fiber provides better characteristics as compared to other approaches and so can be used in highspeed fiber optic communication systems for design of spatially and spectrally transparent media with an increased Raman gain [12, 13].

\section{MODEL OF A SPUN FIBER RAMAN AMPLIFIER WITH A RANDOM BIREFRINGENCE}

Evolution of the signal and pump states of polarization (vectors $\mathbf{S}=\left(S_{1}, S_{2}, S_{3}\right)$ and $\mathbf{P}=\left(P_{1}, P_{2}, P_{3}\right) \quad$ pointing to positions on the Poincaré sphere) is effected by Raman amplification and SOPs interaction and so can be found as follows:

$$
\mathbf{S}(z)=s_{0}(z) G_{\text {ave }}(z) \hat{\mathbf{s}}(z), \quad \mathbf{P}(z)=P_{0}(z) \hat{\mathbf{p}}(z) .
$$

Here $\mathrm{z}$ is the coordinate along the fiber, $\hat{\mathbf{s}}(z)$ and $\hat{\mathbf{p}}(z)$ are unit vector, $G_{\text {ave }}(z)=\exp \left(\int_{0}^{z} g P_{0}\left(z^{\prime}\right) / 2 d z^{\prime}-\alpha_{s} z\right)$ is the averaged Raman gain, $g$ is the Raman gain coefficient, the forward pump power $P_{0}(z)=P_{i n} \exp \left(-\alpha_{p} z\right) ; \alpha_{s}$ and $\alpha_{p}$ are the signal and pump losses, respectively $s_{0}(z)$ is the part of the signal amplitude related to pump-signal SOPs interaction. The part of the Raman gain $\Delta G$ related to polarization evolution due to random birefringence and fiber spinning depends on $s_{0}$ as follows [5-8]:

$$
\Delta G \equiv 10 \log \left(\frac{\left\langle S_{0}(L)\right\rangle}{\left\langle S_{0}(0)\right\rangle} / G_{\text {ave }}\right)=10 \log \left(\frac{\left\langle s_{0}(L)\right\rangle}{\left\langle s_{0}(0)\right\rangle}\right) .
$$


If the input pump and signal SOPs are parallel, the Raman gain takes the maximum value and if the SOPs are orthogonal then Raman gain takes the minimum one [4-8]. The difference in the gain (polarization dependent gain, PDG) and mean-square gain fluctuations (MSGF) $\sigma$ can be found as follows [5-8]

$$
\begin{aligned}
& P D G \equiv 10 \log \left(\left\langle s_{0, \max }(L)\right\rangle /\left\langle s_{0, \text { min }}(L)\right\rangle\right), \\
& \sigma_{\max (\min )}=\sqrt{\left\langle s_{0, \max (\min )}^{2}(L)\right\rangle-\left\langle s_{0, \max (\min )}(L)\right\rangle^{2}} /\left\langle s_{0, \max (\min )}(L)\right\rangle^{\prime}
\end{aligned}
$$

where $\langle\ldots\rangle$ means averaging over the birefringence fluctuations along the fiber. To calculate $s_{0}(L)$, we use vector model of the fiber Raman amplifier for forward pump with accounting for fiber spin profile and random birefringence and neglecting for pump depletion [5]:

$$
\begin{aligned}
& \frac{d \mathbf{s}}{d z}=\frac{g}{2} P_{0}(z) s_{0} \hat{\mathbf{p}}+\left(\boldsymbol{W}_{s}+\boldsymbol{W}_{s}^{(N L)}\right) \times \boldsymbol{s}, \\
& \frac{d \hat{\mathbf{p}}}{d z}=\left(\boldsymbol{W}_{p}+\boldsymbol{W}_{p}^{(N L)}\right) \times \hat{\mathbf{p}},
\end{aligned}
$$

Here $\mathbf{s}=s_{0} \hat{\mathbf{s}}$, and $\boldsymbol{W}_{p}^{(N L)}, \boldsymbol{W}_{s}^{(N L)}$ describes the nonlinear SOP evolution caused by self- and cross-phase modulation (SPM and $\quad \mathrm{XPM} \quad$ ): $\quad \boldsymbol{W}_{p}^{(N L)}=2 \gamma_{p} / 3\left(-2 S_{1},-2 S_{2}, \hat{p}_{3} P_{0}(z)\right)$, $\boldsymbol{W}_{s}^{(N L)}=2 \gamma_{s} / 3\left(-2 \hat{p}_{1} P_{0}(z),-2 \hat{p}_{2} P_{0}(z), S_{3}\right) . \quad$ Kerr coupling constants are $\gamma_{i}=2 \pi n_{2} /\left(\lambda_{i} A_{\text {eff }}\right)(\mathrm{i}=\mathrm{s}, \mathrm{p})$, where $n_{2}$ is the nonlinear Kerr coefficient and $A_{\text {eff }}$ is the effective core area of the fiber. Due to linear birefringence, unit vectors $\hat{\mathbf{s}}$ and $\hat{\mathbf{p}}$ rotate on the Poincaré sphere around the birefringence vector $\boldsymbol{W}_{i}=\left(2 b_{i} \cos \theta\right.$, $\left.2 b_{i} \sin \theta, 0\right)^{T}$ in the same direction, but at different rates $b_{\mathrm{s}}$ and $b_{\mathrm{p}}$ which are birefringence strengths $\left(b_{i}=\pi / L_{\mathrm{b} i}\right.$ where $L_{\mathrm{b} i}$ is the beat length) at signal $\lambda_{\mathrm{s}}$ and pump $\lambda_{p}$ wavelengths. The random birefringence in a single mode fiber (SMF) can be represented in terms of a fixed modulus model (FMM) where the birefringence strength $2 b_{i}$ is fixed and the orientation angle $\theta$ is driven by a white-noise process [1]

$$
\frac{d \theta}{d z}=\beta(z),\langle\beta(z)\rangle=0,\left\langle\beta(z) \beta\left(z^{\prime}\right)\right\rangle=\sigma^{2} \delta\left(z-z^{\prime}\right),
$$

where $\delta(z)$ is a Dirac delta-function, and $\sigma^{2}=2 / L_{\mathrm{c}}\left(L_{c}\right.$ is the birefringence correlation length). Here we neglect the fiber twist and, therefore, the birefringence vector for the spun fiber takes the form of $\boldsymbol{W}_{i, s}=R_{3}[2 A(z)] \boldsymbol{W}_{i}$, where $A(z)$ is the spin profile, and $R_{3}(\gamma)$ represents rotation in the equatorial plane by angle $\gamma$ around the $z$-axis on the Poincare sphere [7]

$$
R_{3}(\gamma)=\left(\begin{array}{ccc}
\cos \gamma & \sin \gamma & 0 \\
-\sin \gamma & \cos \gamma & 0 \\
0 & 0 & 1
\end{array}\right)
$$

We choose the reference frame in the Stokes space in such a way that the local birefringence vector is $\tilde{\mathbf{W}}_{\mathrm{i}, u n}=\left(2 b_{i}, 0,0\right)$ and $\hat{\tilde{\mathbf{p}}}(0)=(1,0,0)$, i.e. oriented along the $X$-axis on the Poincaré sphere. This can be accomplished by a suitable transformation $\tilde{\mathbf{W}}_{i}=\mathbf{R}_{3}^{-1}(\theta+2 A(z)) \mathbf{W}_{\mathrm{i}}, \quad$ and $\quad \tilde{\mathbf{s}}=\mathbf{R}_{3}^{-1}(\theta+2 A(z)) \mathbf{s}$ and $\hat{\tilde{\mathbf{p}}}=\mathbf{R}_{3}^{-1}(\theta+2 A(z)) \hat{\mathbf{p}}$, provided the variable $s_{0}$ and scalar product $x=\hat{\mathbf{p}} \cdot \mathbf{s}$ are invariant under the rotation. In addition, we consider cases which correspond to the maximum and minimum of the Raman gain, viz. $\widetilde{\mathbf{s}}_{\max }(0)=(1,0,0)$ and $\widetilde{\mathbf{S}}_{\min }(0)=(-1,0,0)$. Applying a procedure of averaging over birefringence fluctuations (Stochastic Generator Approach) given in Refs. [1] and [4] to Eqs. (4), we construct the system of equations from which PDG can be calculated with the help of Eqs. (3)

$$
\begin{aligned}
& \frac{d\left\langle s_{0}\right\rangle}{d z^{\prime}}=\varepsilon_{1} \exp \left(-\varepsilon_{2} z^{\prime}\right)\langle x\rangle, \\
& \frac{d\langle x\rangle}{d z^{\prime}}=\varepsilon_{1} \exp \left(-\varepsilon_{2} z^{\prime}\right)\left\langle s_{0}\right\rangle-\varepsilon_{3}\langle y\rangle- \\
& \left(\delta_{p}-\delta_{s}\right) \exp \left(-\varepsilon_{2} z^{\prime}\right)\left\langle\hat{\tilde{p}}_{3}\left(\hat{\tilde{p}}_{2} \tilde{s}_{1}-\hat{\tilde{p}}_{1} \tilde{s}_{2}\right)\right\rangle, \\
& \frac{d\langle y\rangle}{d z^{\prime}}=\varepsilon_{3}\left[\langle x\rangle-\left\langle\hat{\tilde{p}}_{1} \tilde{s}_{1}\right\rangle\right]-2 \alpha\left(z^{\prime}\right)\langle u\rangle-\frac{\langle y\rangle L}{2 L_{c}}+\varepsilon_{4}\left\langle\hat{\tilde{p}}_{2} \tilde{s}_{1}\right\rangle-\varepsilon_{5}\left\langle\hat{\tilde{p}}_{1} \tilde{s}_{2}\right\rangle- \\
& \left(\delta_{p}-\delta_{s}\right) \exp \left(-\varepsilon_{2} z^{\prime}\right)\left(\hat{\tilde{p}}_{3} \hat{\tilde{p}}_{1} \tilde{s}_{3}\right\rangle-\delta_{s} \exp \left(-\varepsilon_{2} z^{\prime}\right)\left\langle\hat{\tilde{p}}_{2}\left(\hat{\tilde{p}}_{2} \tilde{s}_{1}-\hat{\tilde{p}}_{1} \tilde{s}_{2}\right)\right\rangle, \\
& \frac{d\langle u\rangle}{d z^{\prime}}=2 \alpha\left(z^{\prime}\right)\langle y\rangle-\frac{\langle u\rangle}{2}+\left(\delta_{p}-\delta_{s}\right) \exp \left(-\varepsilon_{2} z^{\prime}\right)\left\langle\hat{\tilde{p}}_{3} \hat{\tilde{p}}_{2} \tilde{s}_{3}\right\rangle- \\
& \delta_{s} \exp \left(-\varepsilon_{2} z^{\prime}\right)\left\langle\hat{\tilde{p}}_{1}\left(\hat{\tilde{p}}_{2} \tilde{s}_{1}-\hat{\tilde{p}}_{1} \tilde{s}_{2}\right)\right\rangle,
\end{aligned}
$$

Here $\quad \delta_{p}=2 \gamma_{p} L P_{i n} / 3 \quad \delta_{s}=4 \gamma_{s} L P_{i n} / 3, \quad\langle x\rangle=\left\langle\hat{\tilde{p}}_{1} \tilde{s}_{1}+\hat{\tilde{p}}_{2} \tilde{s}_{2}+\hat{\tilde{p}}_{3} \tilde{s}_{3}\right\rangle$, $\langle y\rangle=\left\langle\hat{\tilde{p}}_{3} \tilde{s}_{2}-\hat{\tilde{p}}_{2} \tilde{s}_{3}\right\rangle,\langle u\rangle=\left\langle\hat{\tilde{p}}_{3} \tilde{s}_{1}-\hat{\tilde{p}}_{1} \tilde{s}_{3}\right\rangle, \alpha\left(z^{\prime}\right)=\partial A\left(z^{\prime}\right) / \partial z^{\prime}$ is the spin rate, $z^{\prime}=z / L, \varepsilon_{1}=g P_{i n} L / 2, \varepsilon_{2}=\alpha_{s} L, \varepsilon_{3}=2 \pi L / L_{b p}\left(\lambda_{s} / \lambda_{p}-1\right)$, $\varepsilon_{4}=(2 \pi L) / L_{b p}, \varepsilon_{5}=(2 \pi L) / L_{b s}$.

In view of $\varepsilon_{3}<<\varepsilon_{4}, \varepsilon_{5}$ (rotation of signal SOP with respect to pump SOP is much slower than SOPs rotation with respect the local birefringence vector $\tilde{\mathbf{W}}_{i}$ ), we can average over the fast oscillations. It can be done by using the transformations $h=\mathbf{R}_{s}(z) \tilde{\mathbf{s}}$ and $f=\mathbf{R}_{p}(z) \hat{\tilde{\mathbf{p}}}$, where

$$
\mathbf{R}_{i}(z)=\left(\begin{array}{ccc}
1 & 0 & 0 \\
0 & \cos \left(2 b_{i} z\right) & \sin \left(2 b_{i} z\right) \\
0 & -\sin \left(2 b_{i} z\right) & \cos \left(2 b_{i} z\right)
\end{array}\right), \quad(i=s, p) .
$$

Using this transformation and averaging over birefringence fluctuations and the fast oscillations, we find the following equation for $\left\langle h_{1} f_{1}\right\rangle$

$$
\frac{d\left\langle h_{1} f_{1}\right\rangle}{d z^{\prime}}=\varepsilon_{1} \exp \left(-\varepsilon_{2} z^{\prime}\right)\left\langle s_{0} f_{1}^{2}\right\rangle-\frac{L}{L_{c}}\left\langle h_{1} f_{1}\right\rangle .
$$

If we choose parameters for Raman amplifier as $P_{i n}=5 \mathrm{~W}$, $L=10 \mathrm{~km}, g=2.3 \mathrm{~dB} W^{1} \mathrm{~km}^{-1}, L_{c}=110 \mathrm{~m}$, we find that $\varepsilon_{1} L_{c} / L=0.15<<1$. Thus, we can neglect the first term on the right-hand side of equation (9). As a result, we find 


$$
\left\langle\hat{\tilde{p}}_{1} \tilde{s}_{1}\right\rangle=\left\langle h_{1} f_{1}\right\rangle=\hat{\tilde{p}}_{1}(0) \widetilde{s}_{1}(0) \exp \left(-z^{\prime} L / L_{c}\right)
$$

In addition, this procedure leads to $\left\langle\hat{\tilde{p}}_{2} \tilde{s}_{1}\right\rangle \rightarrow 0,\left\langle\hat{\tilde{p}}_{1} \tilde{s}_{2}\right\rangle \rightarrow 0$, $\left\langle\hat{\tilde{p}}_{2} \hat{\tilde{p}}_{3} \tilde{s}_{2}\right\rangle \rightarrow 0,\left\langle\hat{\tilde{\tilde{p}}} \hat{\tilde{p}}_{3} \tilde{s}_{2}\right\rangle \rightarrow 0, \quad\left\langle\hat{\tilde{p}}_{3}\left(\hat{\tilde{p}}_{2} \tilde{s}_{1}-\hat{\tilde{p}}_{1} \tilde{s}_{2}\right)\right\rangle \rightarrow 0$ and so selfand cross-phase modulation (SPM and XPM) can be neglected [5-8]. The similar result have been obtained in $[9,11]$ by direct modeling of stochastic equations (4), viz. it was obtained that for the pump power $\mathrm{P}_{\text {in }}<10 \mathrm{~W}$ and PMD parameter $D_{p}>0.01 \mathrm{ps} / \mathrm{km}^{1 / 2} \mathrm{SPM}$ and XPM has no contribution to polarization pulling. Using transformation (8) and averaging over the birefringence fluctuations and fast oscillations we find the following equations from which MSGF $\sigma$ can be found with the help of Eqs. (3):

$$
\begin{aligned}
& \frac{d\left\langle s_{0}^{2}\right\rangle}{d z^{\prime}}=2 \varepsilon_{1} \exp \left(-\varepsilon_{2} z^{\prime}\right)\left\langle s_{0} x\right\rangle, \\
& \frac{d\left\langle s_{0} x\right\rangle}{d z^{\prime}}=\varepsilon_{1} \exp \left(-\varepsilon_{2} z^{\prime}\right)\left(s_{0}^{2}+x^{2}\right)-\varepsilon_{3}\left\langle y s_{0}\right\rangle, \\
& \frac{d\left\langle s_{0} y\right\rangle}{d z^{\prime}}=\varepsilon_{1} \exp \left(-\varepsilon_{2} z^{\prime}\right)\langle x y\rangle+\varepsilon_{3}\left[\left\langle s_{0} x\right\rangle-\left\langle y^{2}\right\rangle-\left\langle s_{0}\right\rangle\left\langle\hat{\tilde{p}}_{1} \tilde{s}_{1}\right\rangle\right]- \\
& 2 \alpha\left(z^{\prime}\right)\left\langle s_{0} u\right\rangle-\frac{\left\langle s_{0} y\right\rangle L}{2 L_{c}}, \\
& \frac{d\left\langle x^{2}\right\rangle}{d z^{\prime}}=2 \varepsilon_{1} \exp \left(-\varepsilon_{2} z^{\prime}\right)\left\langle s_{0} x\right\rangle-2 \varepsilon_{3}\langle x y\rangle, \\
& \frac{d\langle x y\rangle}{d z^{\prime}}=\varepsilon_{1} \exp \left(-\varepsilon_{2} z^{\prime}\right)\left\langle s_{0} y\right\rangle+\varepsilon_{3}\left[\left\langle x^{2}\right\rangle-\langle x\rangle\left\langle\hat{\tilde{p}}_{1} \tilde{s}_{1}\right\rangle\right]- \\
& 2 \alpha\left(z^{\prime}\right)\langle x u\rangle-\frac{\langle x y\rangle L}{2 L_{c}}, \\
& \frac{d\left\langle u s_{0}\right\rangle}{d z^{\prime}}=\varepsilon_{1} \exp \left(-\varepsilon_{2} z^{\prime}\right)\langle x u\rangle+2 \alpha\left(z^{\prime}\right)\left\langle y s_{0}\right\rangle-\frac{\left\langle u s_{0}\right\rangle L}{2 L_{c}}, \\
& \frac{d\langle x u\rangle}{d z^{\prime}}=\varepsilon_{1} \exp \left(-\varepsilon_{2} z^{\prime}\right)\left\langle s_{0} u\right\rangle-\varepsilon_{3}\langle y u\rangle+2 \alpha\left(z^{\prime}\right)\langle x y\rangle-\frac{\langle x u\rangle L}{2 L_{c}}, \\
& \frac{d\langle y u\rangle}{d z^{\prime}}=\varepsilon_{3}\left(\langle x u\rangle-\langle u\rangle\left\langle\hat{\tilde{p}}_{1} \tilde{s}_{1}\right\rangle\right)+2 \alpha\left(z^{\prime}\right)\left(\left\langle y^{2}\right\rangle-\left\langle u^{2}\right\rangle\right)+\frac{L\langle y u\rangle}{2 L_{c}}, \\
& \frac{d\left\langle u^{2}\right\rangle}{d z^{\prime}}=2 \alpha\left(z^{\prime}\right)\langle y u\rangle+\frac{L}{L_{c}}\left(\left\langle y^{2}\right\rangle-\left\langle u^{2}\right\rangle\right), \\
& \frac{d\left\langle y^{2}\right\rangle}{d z^{\prime}}=2 \varepsilon_{3}\left[\langle y x\rangle-\langle y\rangle\left\langle\left(\hat{\tilde{p}}_{1} \tilde{s}_{1}\right\rangle\right]-2 \alpha\left(z^{\prime}\right)\langle y u\rangle-\frac{L}{L_{c}}\left(\left\langle y^{2}\right\rangle-\left\langle u^{2}\right\rangle\right) .\right.
\end{aligned}
$$

To characterize polarization pulling of the signal SOP to the pump SOP we introduce polarization pulling metric $\cos \Phi$ as follows:

$$
\cos \Phi=\langle x\rangle /\left\langle s_{0}\right\rangle .
$$

It is clear that for polarization pulling $\cos \Phi \rightarrow 1$.

To calculate spin induced reduction factor (SIRF) for the case of spun fiber we use the standard model of PMD [3]

$$
\frac{d \boldsymbol{\Omega}}{d z}=\frac{\partial \mathbf{W}_{s}}{\partial \omega}+\mathbf{W}_{s} \times \boldsymbol{\Omega}, \quad \text { SIRF }=\sqrt{\left\langle|\boldsymbol{\Omega}(L)|_{s p}^{2}\right\rangle /\left\langle\left.\boldsymbol{\Omega}(L)\right|_{u n} ^{2}\right\rangle},
$$

where $\left\langle\boldsymbol{\Omega}(L)_{s p}^{2}\right\rangle$ and $\left\langle\boldsymbol{\Omega}(L)_{\text {iun }}^{2}\right\rangle$ are the mean-square differential group delays (DGD) for two orthogonal SOPs in the case of long-length spun fiber and the same fiber without spin, respectively [3]. As follows from [3, 7, 8], after averaging over fluctuations caused by random birefringence, equations for the SIRF take the following form:

$$
\begin{aligned}
& \frac{d \operatorname{SIRF}^{2}}{d z^{\prime}}=\left\langle\hat{\Omega}_{1}\right\rangle, \\
& \frac{d\left\langle\hat{\Omega}_{1}\right\rangle}{d z^{\prime}}=-\left\langle\hat{\Omega}_{1}\right\rangle L / L_{c}+2 \alpha(z)\left\langle\hat{\Omega}_{2}\right\rangle+L / L_{c}, \\
& \frac{d\left\langle\hat{\Omega}_{2}\right\rangle}{d z^{\prime}}=-2 \alpha(z)\left\langle\hat{\Omega}_{1}\right\rangle-\left\langle\hat{\Omega}_{2}\right\rangle L / L_{c}-\varepsilon_{5}\left\langle\hat{\Omega}_{3}\right\rangle, \\
& \frac{d\left\langle\hat{\Omega}_{3}\right\rangle}{d z^{\prime}}=\varepsilon_{5}\left\langle\hat{\Omega}_{2}\right\rangle .
\end{aligned}
$$

In our previous publications, have demonstrated that by combining the short-length fiber with the length $L_{l}$ and without spin, and the long periodically spun fiber with the spin profile $A=A_{0} \sin (2 \pi z / p)\left(A_{0}\right.$ and $p$ are spin amplitude and period), one can mitigate PDG and PMD simultaneously [7, 8]. Optimal parameters $L_{c}, L_{l}$, and $D_{p}^{(1, u n)}$ corresponding to the minimum in SIRF can be found as follows [8]

$$
\sqrt{L_{c}} D_{p}^{(1)}=\frac{1}{4 \pi c\left(1 / \lambda_{p}-1 / \lambda_{s}\right)}, L_{1} \approx 4 \pi L_{c} .
$$

To calculate PDG for the case of a depolarizer application, we use Lin and Agrawal approach [5], viz. transformation of the input pump power $P_{0}$ as $P_{0} \rightarrow D O P \cdot P_{0}$, where DOP is a degree of polarization at the depolarizer output.

\section{RESULTS AND DISCUSSION}

We have used parameters typical for a SMF based distributed fiber Raman amplifier: $L=10 \mathrm{~km}, \alpha_{\mathrm{s}}=0.2 \mathrm{~dB} / \mathrm{km}$, $\lambda_{\mathrm{p}}=1460 \mathrm{~nm}, \lambda_{\mathrm{s}}=1550 \mathrm{~nm}, g=2.3 \mathrm{~dB} \mathrm{~W}^{-1} \mathrm{~km}^{-1}$, and $P=5 \mathrm{~W}$. First, we have calculated PMD values for the two-section fiber with the help of equation (15). In view of Eq. (16), one of the parameters $D_{\mathrm{p}}^{(1, \text { un })}$ or $L_{\mathrm{c}}$ can be chosen arbitrary, so we picked $D_{\mathrm{p}}^{(1)}=0.1 \mathrm{ps} \cdot \mathrm{km}^{-1 / 2}$ and $L_{c}=5 \mathrm{~m}$. Next, we have calculated PDG, MSGF, SIRF from equations (3) and averaged equations (7), (12) and (15) for the cases of periodically spun onesection fiber, two-section fiber and application of pump depolarizer. The results are shown in Figs. $1(\mathrm{a}-\mathrm{b})$. The results in Table 1 show optimal values of PDG, MSGF which have been chosen at the minimum values of SIRF.

TABLE I

ONE-SECTION, TWO-SECTION RAMAN POLARIZER AND APPLICATION OF DEPOLARIZER (FROM FIG. 1 (A,B))

\begin{tabular}{cccc}
\hline \hline Specifications & $\begin{array}{l}\text { One-section } \\
\text { Raman polarizer }\end{array}$ & $\begin{array}{c}\text { Two-section } \\
\text { Raman polarizer }\end{array}$ & $\begin{array}{c}\text { Application of } \\
\text { Depolarizer }\end{array}$ \\
\hline PDG, dB & 20 & 0.23 & 4.5 \\
Min SIRF & 0.005 & 0.08 & 0.05 \\
$\sigma_{\operatorname{maxG}}, \%$ & 5 & 54.5 & 3.3 \\
$\sigma_{\operatorname{minG}}, \%$ & 95 & 60.7 & 7.3
\end{tabular}


Finally, we calculated SIRF and maximum/minimum PDG and $\sigma$ for a periodically spun fiber in the case of application of a depolarizer $(\mathrm{DOP}=10 \%)$ with the help of Eqs $(15)$ and accounting for changing the parameter in Eqs. (9) as follows: $\varepsilon_{1} \rightarrow \varepsilon_{1} D O P$ (Fig. $1(\mathrm{a}, \mathrm{b})$ ). As follows from [14], temperature fluctuations can lead to an increased DOP for the input pump wave of $10-15 \%$. In view of this, it can lead to an increased PDG value of $4.5 \mathrm{~dB}$ and gain fluctuations to 3.3 and $7.3 \%$ (thick solid lines in Fig1 (a) and thin and thick solid lines in Fig. 1 (b)).

a)

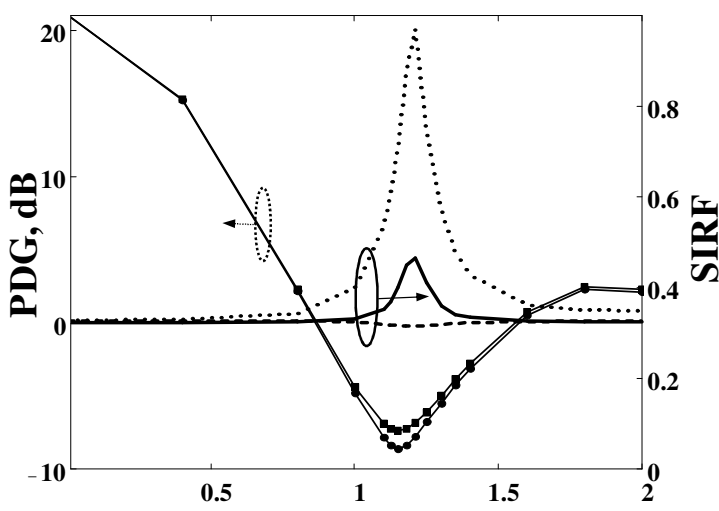

Amplitude of fiber spinning $A_{0}$, rad

b)

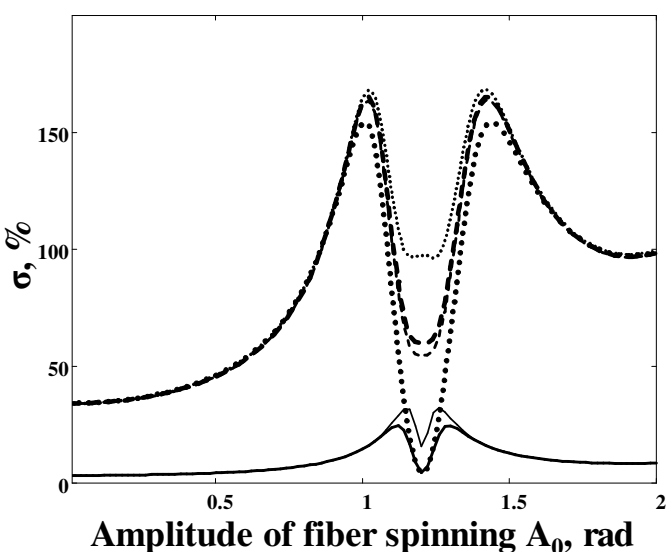

Figure 1 Polarization dependent gain PDG, spin induced reduction factor SIRF (a) and mean-square gain fluctuations $\sigma$ (b) as function of a fiber spinning amplitude $\mathrm{A}_{0}$. Dotted line and circles: periodically spun one-section fiber, dashed line and squares: two-section fiber, solid line: application of depolarizer with $\mathrm{DOP}=10 \%$. Pump input SOP $(1,0,0)$, signal input SOPs ($1,0,0)$ (thin lines) and $(1,0,0)$ (thick lines). Parameters: $L=10 \mathrm{~km}, \alpha_{\mathrm{s}}=0.2$ $\mathrm{dB} / \mathrm{km}, \lambda_{\mathrm{p}}=1460 \mathrm{~nm}, \lambda_{\mathrm{s}}=1550 \mathrm{~nm}, \mathrm{~g}=2.3 \mathrm{~dB} \mathrm{~W}^{-1} \mathrm{~km}^{-1}, \mathrm{P}=5 \mathrm{~W}, \mathrm{D}_{\mathrm{p}}{ }^{(1)}=0.1$ $\mathrm{ps} \cdot \mathrm{km}^{-1 / 2} \mathrm{~L}_{\mathrm{c}}=5 \mathrm{~m}$

As follows from Fig.1 and Table 1, application of periodically spun fiber results in low PMD (SIRF) value. The fiber becomes more isotropic and so difference in the gain values for cross-polarized signal SOPs have maximum, and PDG increases up to $20 \mathrm{~dB}$. In addition, gain fluctuations are high for the case of input signal SOP orthogonally polarized to the pump SOP. As follows from Fig. 1 (c) (thin lines), such a fiber works as a Raman polarizer but it requires higher power for pulling signal input SOP cross-polarized to the pump input SOP.

By using two-section technique it is possible to suppress PDG further down to $0.23 \mathrm{~dB}$. Although this approach suffers from high gain fluctuations it is nevertheless very promising for further development in the context of application as a Raman polarizer. As follows from Fig. 1 (c) (solid lines), twosection approach provides almost equal polarization pulling for cross-polarized input signal SOPs and so results in almost equal gain fluctuations and low PDG.

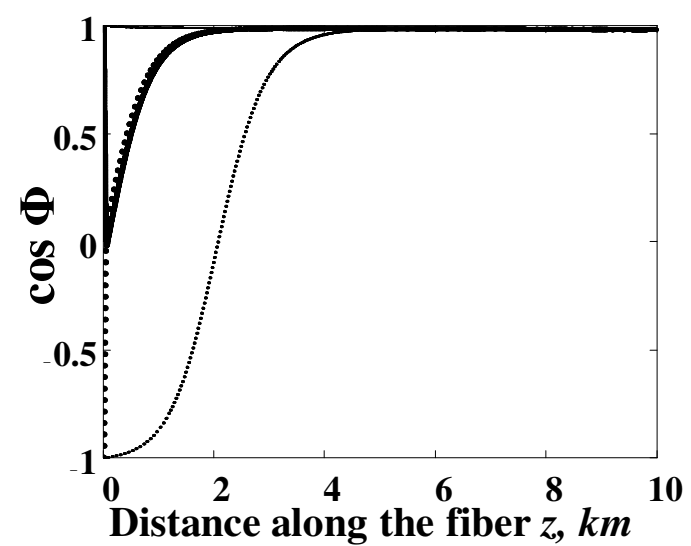

Figure 2 Polarization pulling metrics $\cos \Phi$ as a function of distance along the fiber $\mathrm{z}$. One section of periodically spun fiber (dotted lines), two-section fiber (dotted line). Pump input SOP $(1,0,0)$, signal input SOPs $(-1,0,0)$ (thin lines) and $(1,0,0)$ (thick lines). Parameters: $L=10 \mathrm{~km}, \alpha_{\mathrm{s}}=0.2 \mathrm{~dB} / \mathrm{km}$, $\lambda_{\mathrm{p}}=1460 \mathrm{~nm}, \lambda_{\mathrm{s}}=1550 \mathrm{~nm}, \mathrm{~g}=2.3 \mathrm{~dB} \mathrm{~W}^{-1} \mathrm{~km}^{-1}, \mathrm{P}=3 \mathrm{~W}, \mathrm{D}_{\mathrm{p}}{ }^{(1)}=0.1 \mathrm{ps} \cdot \mathrm{km}^{-1 / 2}$ $\mathrm{L}_{\mathrm{c}}=5 \mathrm{~m}, \mathrm{~A}_{0}=1.21 \mathrm{rad}$.

\section{CONCLUSION}

Applying the vector model of a fiber Raman amplifier accounting for fiber spin profile and random birefringence, we provide comparative analysis of different schemes for suppression of polarization dependent gain and polarization mode dispersion. We have shown that application of a twosection approach results in suppression of PDG down to 0.23 $\mathrm{dB}$ and SIRF down to 0.08. The first section is short, it has specified parameters (length, PMD, correlation length) and no spin. The second section is a long periodically spun fiber. In addition, two-section fiber provides almost equal polarization pulling for cross-polarized input signal SOPs and so can be used as a new type of a fiber optic Raman polarizer.

S. Sergeyev acknowledges financial support from the European Union program FP7 PEOPLE-2009-IEF (grant 253297).

\section{REFERENCES}

[1] P. K. A. Wai, C. R. Menyuk, "Polarization mode dispersion, decorrelation and diffusion in optical fibers with randomly varying birefringence," J. Lightwave Technol., vol. 14, 148-157 (1996).

[2] D. A. Nolan, X. Chen, M.-J. Li, "Fibers with low polarization-mode dispersion, “ J. Lightwave Technol., vol. 22, 1066-1077 (2004).

[3] A. Galtarossa, L. Palmieri, A. Pizzinat, B. S. Marks, and C. R. Menyuk, "An analytical formula for the mean differential group delay of 
randomly-birefringent spun fibers," J. Lightwave Technol. 21, 16351643 (2003).

[4] R. H. Stolen, "Polarization effects in fiber Raman and Brillouin Lasers", IEEE J. Quant. Electron., vol. 15, 1157-1160 (1979).

[5] Q. Lin, G. P. Agrawal, "Vector theory of stimulated Raman scattering and its application to fiber-based Raman amplifiers, “J. Opt. Soc. Am. $B$, vol. 20, pp. 1616-1631 (2003).

[6] S. Sergeyev, S. Popov, and A. T. Friberg, "Modeling polarizationdependent gain in fiber Raman amplifiers with randomly varying birefringence," Opt. Comm., vol. 262, 114-119 (2006).

[7] S. Sergeyev, S. Popov, A. T. Friberg, "Spun fiber Raman amplifiers with reduced polarization impairments", Opt. Express, vol. 16, 1438014389 (2008).

[8] S. Sergeyev, S. Popov, A. T. Friberg, "Virtually Isotropic Transmission Media With Fiber Raman Amplifier, IEEE J. Quantum Electron.," vol. 46, 1492-1497 (2010).

[9] M. Martinelli, M. Cirigliano, M. Ferrario, L. Marazzi, and P. Martelli, "Evidence of Raman-induced polarization pulling," Opt. Express 17, 947-955 (2009)

[10] V. V. Kozlov, J. Nuño, J. D. Ania-Castañón, and S. Wabnitz, "Theory of fiber optic Raman polarizers," Opt. Lett. 35, 3970-3972 (2010).

[11] L. Ursini, M. Santagiustina, and L. Palmieri, "Raman Nonlinear Polarization Pulling in the Pump Depleted Regime in Randomly Birefringent Fibers," IEEE Photon. Technol. Lett., 23, 254-256 (2011).

[12] J. D. Ania-Castanon, V. Karalekas, P. Harper, and S. K. Turitsyn, "Simultaneous spatial and spectral transparency in ultralong fiber lasers", Phys. Rev. Lett., vol. 101, 123903-123907 (2008).

[13] S. A. Babin, V. Karalekas, E. V. Podivilov, V. K. Mezentsev, P. Harper, J. D. Ania-Castanon, and S. K. Turitsyn, "Turbulent broadening of optical spectra in ultralong Raman fiber lasers", Phys. Rev. A, vol. 77, 033803-1-5 (2008).

[14] H. Kazami, S. Matsushita, Y. Emori, T. Murase, M. Tsuyuki, K. Yamamoto, H. Matsuura, S. Namiki, and T. Shiba, "Development of a crystal-type depolarizer", Furakawa Review, vol. 23, $44-47$ (2003).

Sergey Sergeyev received the M.Sc. and Ph.D. degrees in Physics from Belarusian State University (BSU), Minsk, in 1985 and 1991, respectively. He was with the Department of Physics, BSU, from 1985 to 1999. From 1999 to 2005, he worked in Sweden at Ericsson AB, Royal Institute of Technology, and Acreo AB. From 2005 to 2010, he worked at Optics Research Group (ORG), Waterford Institute of Technology, Waterford, Ireland as a Senior Researcher/Project Manager. Since 2010 he is working as a Marie Curie Research Fellow at Photonics Research Group, Aston University, Birmingham, UK. He has coauthored over 90 journal and conference papers in the areas of fiber optics, laser physics, and spectroscopy.

Sergei Popov received M.Sc. in El. Eng. (1987, Moscow Institute of Physics and Technology) and Comp. Sc. (1989, Air Force Engineering Academy), both in Moscow, Russia. He holds Ph.D. degree in Applied Physics (1998) from Helsinki University of Technology (Espoo, Finland). Dr. Popov pursued his research career in General Physics Institute (Moscow, Russia), Ericsson Telecom AB and Acreo AB (both in Stockholm, Sweden). Currently, he is Associate Professor at Royal Institute of Technology (Stockholm, Sweden); and contributed to over 100 journal and conference papers on laser physics, diffractive and fiber optics, as well as nano-photonics. 